\title{
Engineering of skeletal muscle tissue using myoblast-seeded bovine pericardium for reconstruction of abdominal wall defect in a rabbit model.
}

\begin{abstract}
A novel tissue engineered construct was used to engineer skeletal muscle tissue for reconstruction of abdominal wall defects, which is a common challenge to surgeons, due to insufficient autogenous tissue. Myoblasts were isolated from soleus muscle fibers, seeded onto the scaffold and cultivated in vitro for 5 days. Full-thickness abdominal wall defects (3 $\times 4 \mathrm{~cm}$ ) were created in 18 male New Zealand white rabbits and randomly divided into two equal groups ( $\mathrm{n}=9$ each). The defects of the first group were repaired with myoblast seeded bovine pericardium (treatment group) whereas the second group involved non-seeded bovine pericardium (control group). Three animals were sacrificed at 7, 14, and 30 days postimplantation from each group and the explanted specimens were subjected to macroscopic, light, fluorescence and electron microscopic analysis. In each case, the tissue engineered construct was thicker from deposition of newly formed collagen with neo-vascularisation, than the control group. Most importantly, multinucleated myotubes and myofibers were only detected in the treatment group. Therefore, this study demonstrates that myoblast-seeded bovine pericardium construct can provide a structural replacement for severe and large abdominal wall defects with profound regeneration of skeletal muscle tissues. (C) (2010) Trans Tech Publications, Switzerland.
\end{abstract}

Keyword: Abdominal wall defect; Bovine pericardium; Cell seeding; Muscle; Tissue engineering. 